\title{
GMR
}

\section{miR-367 promotes uveal melanoma cell proliferation and migration by regulating PTEN}

\author{
J.W. Ling', P.R. Lu ${ }^{2}$, Y.B. Zhang ${ }^{1}$, S. Jiang ${ }^{1}$ and Z.C. Zhang ${ }^{1}$ \\ ${ }^{1}$ Department of Ophthalmology, Third Hospital of Zhangjiagang, Zhangjiagang, \\ China \\ ${ }^{2}$ Department of Ophthalmology, First Affiliated Hospital of SooChow University, \\ SooChow, China \\ Corresponding author: Z.C. Zhang \\ E-mail: zhangzcsy@163.com \\ Genet. Mol. Res. 16 (3): gmr16039067 \\ Received August 10, 2016 \\ Accepted October 31, 2016 \\ Published August 17, 2017 \\ DOI http://dx.doi.org/10.4238/gmr16039067
}

Copyright $(2017$ The Authors. This is an open-access article distributed under the terms of the Creative Commons Attribution ShareAlike (CC BY-SA) 4.0 License.

ABSTRACT. We aimed to investigate the biological role of miR367 in uveal melanoma cell growth and migration, and the underlying mechanism responsible. Quantitative real-time polymerase chain reaction was performed to evaluate miR-367 expression in uveal melanoma tissue samples and cell lines. A miR-367 mimic, miR-367 inhibitor, and negative control oligonucleotide were transfected into these cells to investigate the function of this microRNA. In addition, the role of PTEN in miR-367-mediated uveal melanoma cell growth and migration was evaluated. miR-367 was significantly upregulated in uveal melanoma cells and tissue samples (both $\mathrm{P}<0.01$ ). Its inhibition suppressed the proliferation, cell cycle transition, and migration of such cells, and increased levels had the opposite effect. PTEN was confirmed to be a target gene of miR-367. More importantly, co-transfection with a PTEN construct lacking the 3'-untranslated region mitigated miR-367 mimic-induced promotion of uveal melanoma cell proliferation and migration. In summary, miR-367 was found to be upregulated in this

Genetics and Molecular Research 16 (3): gmr16039067 
malignancy, and may promote uveal melanoma cell proliferation and migration, at least in part by regulating PTEN.

Key words: Uveal melanoma; miR-367; PTEN; Cell proliferation; Cell migration

\section{INTRODUCTION}

Uveal melanoma is the most common primary intraocular malignancy and is associated with a high incidence of systemic metastasis, a key characteristic of this disease (Yousef and Alkilany, 2015). The liver is the principal organ involved in distant metastasis of this cancer, followed by the lungs and soft tissue. At present, no effective treatment strategy exists for metastatic uveal melanoma, and the long-term survival of patients has not significantly improved over the last three decades (Buder et al., 2013). Further understanding of the molecular events contributing to uveal melanoma metastasis will facilitate the development of new therapeutic methods and improve patient outcome.

First identified in the early 1990s, microRNAs (miRNAs) are small regulatory RNA sequences serving important biological functions (Bartel, 2009). Since miRNAs themselves do not encode proteins, their functions rely on the negative regulation of target genes. Many well-known oncogenes and tumor-suppressor genes have been confirmed to be direct targets of various miRNAs, the crucial role of which in cancer development has been widely recognized (Shenouda and Alahari, 2009; Kanekura et al., 2016; Lee et al., 2016). miR-144, miR-34a, and miR-32 inhibit uveal melanoma cell proliferation and migration by regulating c-Met and EZH2 (Yan et al., 2009; Sun et al., 2015a; Ma et al., 2016), whereas miR-454 functions as an oncogene due to its effect on PTEN (Sun et al., 2015b). Achberger et al. (2014) reported that uveal melanoma patients with and without metastasis exhibit different plasma miRNA expression patterns. However, the study of the role of miRNAs in uveal melanoma is still in its infancy.

miR-367 has recently been identified as a novel tumor-related miRNA in colon cancer (Chae et al., 2013), breast cancer (Zhang et al., 2011), medulloblastoma (Kaid et al., 2015), esophageal squamous cell carcinoma (ESCC; Sun et al., 2016), gastric cancer (Bin et al., 2015), osteosarcoma (Wang et al., 2016), hepatocellular carcinoma (Meng et al., 2016), glioma (Guan et al., 2015), pancreatic ductal adenocarcinoma (Zhu et al., 2015), non-small cell lung cancer (NSCLC; Campayo et al., 2013), and cervical carcinoma (Cai et al., 2013). Modulation of miR-367 expression can affect several aspects of cancer cell behavior, including proliferation, apoptosis, invasion, and epithelial-to-mesenchymal transition. However, the role of miR367 in uveal melanoma, if any, has not been reported. In this study, we measured miR-367 expression in uveal melanoma tissues and cell lines, and experimentally demonstrated that this miRNA can promote the growth and migration of such cells by regulating PTEN expression.

\section{MATERIAL AND METHODS}

\section{Tissue specimens, cell culture, and transfection}

This study was approved by the hospital Ethics Committee. Surgical specimens, including uveal melanoma and paired non-tumorous tissues, were obtained from 28 patients having received no treatment prior to surgery, and stored at $-80^{\circ} \mathrm{C}$ until needed. All patients provided their written informed consent.

Genetics and Molecular Research 16 (3): gmr16039067 
Uveal melanoma cell lines (M17, M23, MUM-2B, and C918) and normal uveal melanocytes (um95) were obtained from the American Type Culture Collection (Manassas, VA, USA), and cultured at $37^{\circ} \mathrm{C}$ in a humidified atmosphere in Dulbecco's modified Eagle's medium (DMEM; Corning cellgro, Manassas, VA, USA) containing 10\% fetal bovine serum (v/v; FBS; Gibco, Gaithersburg, MD, USA).

A miR-367 mimic, miR-367 inhibitor (anti-miR-367), and scrambled negative control (NC) sequence were synthesized by RiboBio (Guangzhou, China). The NC sequence was not homologous to any region of the human genome. Uveal melanoma cells were seeded on six-well plates $24 \mathrm{~h}$ before being transiently transfected using Lipofectamine 2000 (Life Technologies, Carlsbad, CA, USA). To determine whether PTEN is involved in miR-367mediated uveal melanoma progression, the expression vector pcDNA3.1-PTEN [lacking the PTEN 3'-untranslated region (UTR)] was obtained from JRDUN Biotechnology (Shanghai, China) and co-transfected with the miR-367 mimic into uveal melanoma cells using Lipofectamine 2000. Cells were used in experiments $48 \mathrm{~h}$ after transfection. All experiments involving cells were repeated three times.

\section{RNA extraction and quantitative real-time polymerase chain reaction (qRT-PCR)}

An RNeasy Mini Kit (QIAGEN, Valencia, CA, USA) was used to extract total RNA, $1 \mu \mathrm{g}$ of which was reverse transcribed and polyadenylated using Bulge-Loop miRNA-specific reverse transcription primers (RiboBio). The products of reverse transcription were then subjected to qRT-PCR using Bulge-Loop primers and SYBR Premix Ex Taq II (TaKaRa, Shiga, Japan) on a 7900HT Fast RT-PCR System (Applied Biosystems, Foster City, CA, USA). The following cycling conditions were used: $95^{\circ} \mathrm{C}$ for $10 \mathrm{~min}$, then 40 cycles of $95^{\circ} \mathrm{C}$ for $15 \mathrm{~s}$ and $60^{\circ} \mathrm{C}$ for $60 \mathrm{~s}$. Relative miR-367 expression was normalized to that of U6 using the $2^{-\Delta \mathrm{Ct}}$ method.

\section{Cell proliferation analysis}

The Cell Counting Kit-8 (CCK-8) assay (Dojindo, Tokyo, Japan) was used to assess cell proliferation. After transfection, uveal melanoma cells were cultured on 96-well plates $\left(5 \times 10^{3}\right.$ cells/well) at $37^{\circ} \mathrm{C}$ for 1,2 , or 3 days. At each time point, $8 \mu \mathrm{L}$ CCK- 8 solution was added to each well and incubated with cells for $90 \mathrm{~min}$. Absorbance was then read at $450 \mathrm{~nm}$ using a microplate reader (SpectraMax M2; Molecular Devices, Sunnyvale, CA, USA).

\section{Cell cycle assay}

Cells were fixed with $75 \%$ ethanol at $-20^{\circ} \mathrm{C}$ overnight and treated with $100 \mathrm{ng} / \mathrm{mL}$ propidium iodide (Thermo Fisher Scientific, Waltham, MA, USA) for 15 min in the dark. Cell cycle phase distribution was assessed by flow cytometry using the FlowJo software (Tree Star, Ashland, OR, USA).

\section{Cell migration assay}

Transfected cells were serum starved for $12 \mathrm{~h}$ before being transferred to the upper chambers of 24-well Transwell plates (8-mm pore size; Corning, Corning, NY, USA) in serum-

Genetics and Molecular Research 16 (3): gmr16039067 
free DMEM $\left(1 \times 10^{5}\right.$ cells $\left./ 500 \mu \mathrm{L}\right)$. The lower chambers were filled with DMEM supplemented with $10 \%$ FBS. Cells were allowed to migrate for $24 \mathrm{~h}$. After incubation, cells having migrated to the lower membrane surface were stained with $0.1 \%$ crystal violet and counted.

\section{Luciferase reporter assay}

The PTEN 3'-UTR containing the wild-type (wt) or a mutant (mt) miR-367 target site was cloned into the pGL3 luciferase reporter vector. Cells were seeded on a 24-well plate (1 x $10^{5}$ cells/well) and allowed to settle for $24 \mathrm{~h}$. Transfection with the pGL3-wt-PTEN or pGL3mt-PTEN plasmid, pRL-TK Renilla luciferase reporter, and miR-367 mimic or NC sequence was then performed using Lipofectamine 2000. A Dual-Luciferase Reporter Assay System (Promega, Madison, WI, USA) was used to measure luciferase activity $48 \mathrm{~h}$ after transfection.

\section{Western blot analysis}

Total cell protein was isolated using radioimmunoprecipitation assay buffer containing $1 \mathrm{X}$ protease inhibitors. Protein $(40 \mu \mathrm{g})$ was loaded on $12 \%$ gels for sodium dodecyl sulfatepolyacrylamide gel electrophoresis, and transferred to polyvinylidene fluoride membranes (Bio-Rad, Richmond, CA, USA). Membranes were blocked with 3\% bovine serum albumin in Tris-buffered saline-Tween 20 (TBST), and probed with anti-PTEN (diluted 1:1000; Abcam, Cambridge, MA, USA) and anti-GAPDH (diluted 1:2000; Santa Cruz Biotechnology, Dallas, TX, USA) primary antibodies. Subsequently, membranes were washed three times in TBST and exposed to horseradish peroxidase-conjugated secondary antibodies. Proteins were then visualized using enhanced chemiluminescence reagents.

\section{Statistical analysis}

All data were analyzed using SPSS version 16.0 (SPSS Inc., Chicago, IL, USA). The Student $t$-test was used to evaluate differences between groups, with $\mathrm{P}$ values $<0.05$ being considered significant.

\section{RESULTS}

\section{miR-367 is increased in uveal melanoma cells and tissues}

We found that miR-367 expression was upregulated in uveal melanoma cells compared with normal uveal melanocytes $(\mathrm{P}<0.01$; Figure 1A). In comparison with normal uveal specimens, we also observed increased miR-367 levels in 28 human uveal melanoma samples $(\mathrm{P}<0.01$; Figure 1B).

\section{miR-367 overexpression promotes uveal melanoma cell proliferation and migration}

Of the four uveal melanoma cell lines used in our study, MUM-2B and M23 cells exhibited relatively high and low baseline expression of miR-367, respectively. Therefore, we transfected the former with anti-miR-367 and the latter with a miR-367 mimic. Using CCK-8 assays, proliferation of MUM-2B cells transfected with anti-miR-367 was observed 
to be suppressed compared to that of those transfected with the NC sequence (Figure 2A). The proportion of MUM-2B cells in the G0/G1 phase increased as a result of anti-miR-367 transfection (Figure 2B). Moreover, anti-miR-367 reduced MUM-2B cell migration (Figure 2C). Conversely, the miR-367 mimic enhanced the proliferation, cell cycle transition, and migration of M23 cells (Figure 3A-C). More importantly, co-transfection with a PTEN construct lacking the 3'-UTR attenuated the miR-367 mimic-induced promotion of M23 cell proliferation and migration (Figure 3A-C).
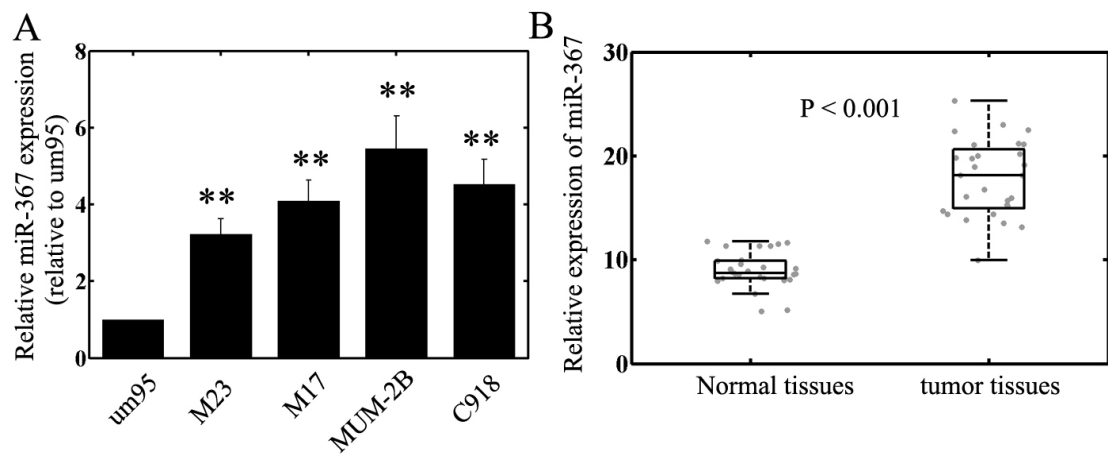

Figure 1. Relative miR-367 expression in uveal melanoma tissues and cell lines. A. Increased miR-367 expression in uveal melanoma cell lines compared to um95 normal uveal melanocytes. $* * \mathrm{P}<0.01$. B. miR-367 was upregulated in uveal melanoma specimens $(\mathrm{N}=28)$ compared to paired non-tumorous tissues.

A
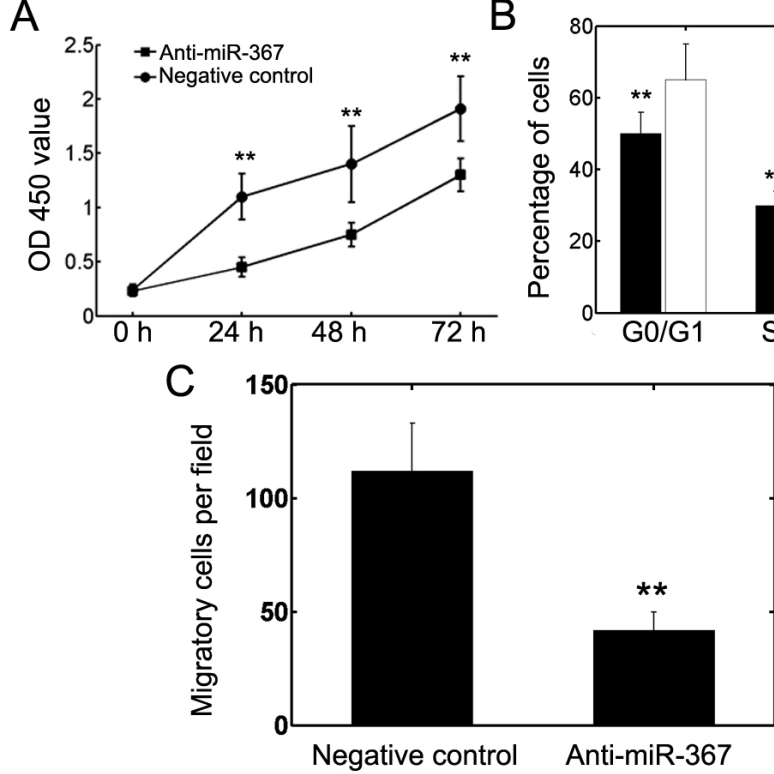

B

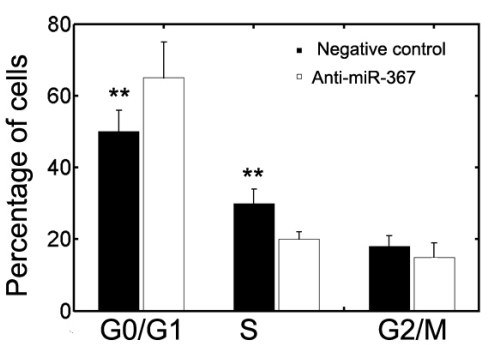

Figure 2. Inhibition of miR-367 suppressed MUM-2B cell proliferation and migration. A. A Cell Counting Kit- 8 assay was performed to examine MUM-2B cell proliferation after transfection with anti-miR-367 or a negative control sequence. ${ }^{* *} \mathrm{P}<0.01$. OD $450=$ optical density at $450 \mathrm{~nm}$. B. Cell cycle analysis showed that the proportion of MUM$2 \mathrm{~B}$ cells in the G0/G1 phase increased due to anti-miR-367 transfection. ${ }^{* * P}<0.01$. C. A Transwell migration assay was performed on MUM-2B cells transfected with anti-miR-367 or a negative control sequence. ${ }^{* *} \mathrm{P}<0.01$. 


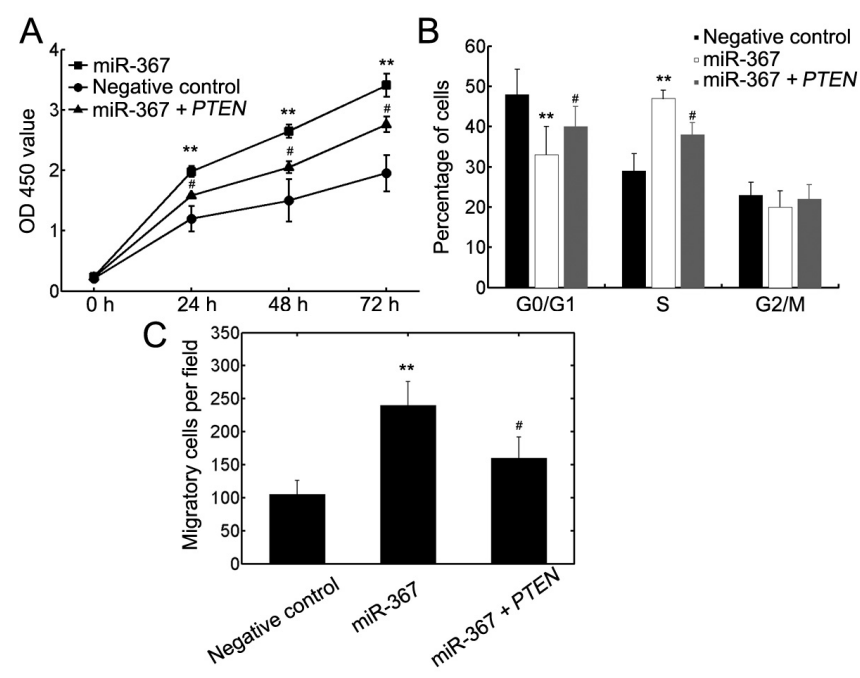

Figure 3. miR-367 enhanced M23 cell proliferation and migration, and co-transfection with PTEN partly negated these effects. A. A Cell Counting Kit-8 assay was performed to examine M23 cell proliferation after transfection with a miR-367 mimic and PTEN. **P $<0.01$ vs negative control; ${ }^{*} \mathrm{P}<0.05$ ss miR-367. OD $450=$ optical density at $450 \mathrm{~nm}$. B. Cell cycle analysis showed that the proportion of M23 cells in the G0/G1 phase decreased as a result of miR-367 mimic transfection, and co-transfection with PTEN partially reversed this outcome. ${ }^{*} * \mathrm{P}<0.01 v s$ negative control; ${ }^{*} \mathrm{P}<0.05$ vs miR-367. C. A Transwell migration assay was performed on $\mathrm{M} 23$ cells transfected with a miR-367 mimic and PTEN. **P $<0.01$ vs negative control; ${ }^{*} \mathrm{P}<0.05$ vs miR-367.

\section{PTEN is a target gene of miR-367}

Figure 4A shows the miR-367-binding site in the 3'-UTR of PTEN. We observed reduced PTEN protein levels in response to increased miR-367 presence in M23 cells (Figure 4B). Furthermore, the relative luciferase activity of cells transfected with pGL3-wt-PTEN was significantly diminished by co-transfection with the miR-367 mimic. However, the latter had no significant effect on the luciferase activity of pGL3-mt-PTEN-transfected cells (Figure 4C), demonstrating that miR-367 may regulate PTEN expression by directly targeting its 3'-UTR.

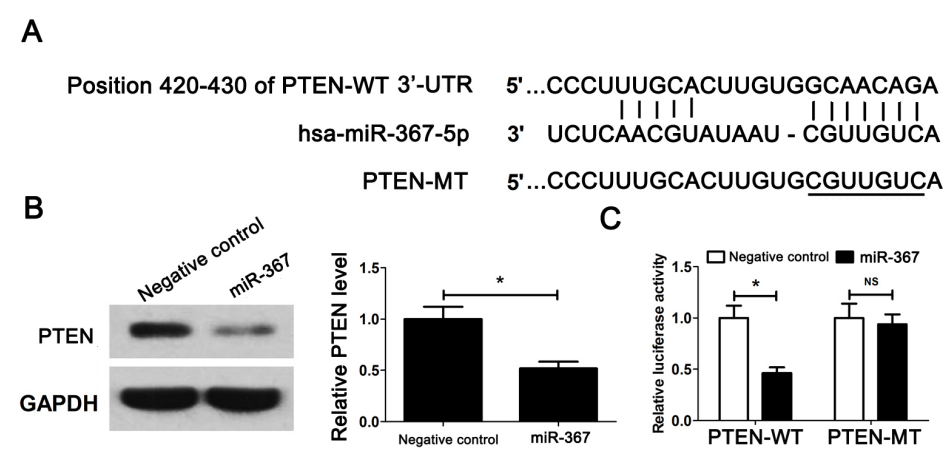

Figure 4. $P T E N$ is a direct target of miR-367. A. Predicted miR-367 target sequence in the 3 '-untranslated region (UTR) of PTEN. WT = wild type; MT = mutant. B. miR-367 upregulation in M23 cells was associated with decreased PTEN protein levels. $* \mathrm{P}<0.05$. C. Analysis of the relative luciferase activities of PTEN-WT and PTENMUT reporter plasmids. $* \mathrm{P}<0.05 ; \mathrm{NS}=$ not significant. 


\section{DISCUSSION}

Recent studies have linked miRNA dysregulation to cancer development. In the present study, we revealed miR-367 to be upregulated in uveal melanoma cells and tissues. Further functional assays indicated that increased miR-367 levels promoted uveal melanoma cell proliferation and migration, and that these effects could be partly attenuated by PTEN overexpression.

Aberrant miR-367 expression has been reported in many cancers. Levels of this miRNA are heightened in tumor tissues and serum samples from ESCC patients, and decrease following esophagectomy and chemotherapy (Sun et al., 2016). High serum miR367 concentration correlates closely with poor tumor differentiation, advanced clinical stage, lymph node metastasis, and reduced overall survival in this malignancy (Sun et al., 2016). Moreover, miR-367 downregulation inhibits the growth and cell cycle transition of ESCC cells (Sun et al., 2016). In NSCLC, elevated miR-367 levels are associated with shorter postoperative relapse-free periods. miR-367 also enhances hepatocellular carcinoma cell proliferation and invasion by regulating PTEN expression (Meng et al., 2016), and promotes pancreatic cancer invasion in vitro and metastasis in vivo through targeting of the Smad7/ TGF- $\beta$ signaling pathway (Zhu et al., 2015). In addition, this miRNA suppresses Adriamycininduced apoptosis of osteosarcoma cells by targeting KLF4 (Wang et al., 2016). However, in contrast to these oncogenic characteristics, miR-367 is downregulated in gastric cancer (Bin et al., 2015), glioma (Guan et al., 2015), and cervical carcinoma (Cai et al., 2013). Reduced miR-367 expression in gastric cancer tissues correlates with tumor metastasis and advanced tumor-node-metastasis stage (Bin et al., 2015), and its overexpression has been shown to inhibit gastric cancer cell invasion via direct suppression of Rab23 (Bin et al., 2015). Ectopic expression of miR-367 restricts proliferation and blocks the G1/S transition of cervical cancer cells through regulation of AKT1 (Cai et al., 2013), and low miR-367 level is an independent indicator of unfavorable prognosis for glioma patients (Guan et al., 2015). Thus, in different cancers, miR-367 acts as either an oncogene or a tumor-suppressor, and its effects in other malignancies warrant further study.

PTEN is a well-known antioncogene, and exerts a tumor-suppressive effect during uveal melanoma pathogenesis (Abdel-Rahman et al., 2006; Sun et al., 2015a). Moreover, loss of PTEN expression is associated with shortened disease-free survival of uveal melanoma patients (AbdelRahman et al., 2006). Our study confirmed that PTEN is a direct target of miR-367. We revealed for the first time that this miRNA suppresses PTEN expression by binding directly to the 3'-UTR, thereby accounting for its oncogenic properties in uveal melanoma. It is now understood that one miRNA can regulate multiple targets. The fact that restoration of PTEN expression did not entirely reverse the effects of miR-367 demonstrates the existence of other target genes involved in miR-367-mediated uveal melanoma cell proliferation and migration.

In conclusion, our study showed that miR-367 was upregulated in uveal melanoma, and it may promote the proliferation and migration of cells of this malignancy, at least in part, by regulating PTEN. Hence, miR-367 could be considered as a potential therapeutic target for the treatment of this disease.

\section{Conflicts of interest}

The authors declare no conflict of interest.

Genetics and Molecular Research 16 (3): gmr16039067 


\section{REFERENCES}

Abdel-Rahman MH, Yang Y, Zhou XP, Craig EL, et al. (2006). High frequency of submicroscopic hemizygous deletion is a major mechanism of loss of expression of PTEN in uveal melanoma. J. Clin. Oncol. 24: 288-295. http://dx.doi. org $/ 10.1200 / \mathrm{JCO} .2005 .02 .2418$

Achberger S, Aldrich W, Tubbs R, Crabb JW, et al. (2014). Circulating immune cell and microRNA in patients with uveal melanoma developing metastatic disease. Mol. Immunol. 58: 182-186. http://dx.doi.org/10.1016/j. molimm.2013.11.018

Bartel DP (2009). MicroRNAs: target recognition and regulatory functions. Cell 136: 215-233. http://dx.doi.org/10.1016/j. cell.2009.01.002

Bin Z, Dedong H, Xiangjie F, Hongwei X, et al. (2015). The microRNA-367 inhibits the invasion and metastasis of gastric cancer by directly repressing Rab23. Genet. Test. Mol. Biomarkers 19: 69-74. http://dx.doi.org/10.1089/ gtmb.2014.0210

Buder K, Gesierich A, Gelbrich G and Goebeler M (2013). Systemic treatment of metastatic uveal melanoma: review of literature and future perspectives. Cancer Med. 2: 674-686. http://dx.doi.org/10.1002/cam4.133

Cai N, Wang YD and Zheng PS (2013). The microRNA-302-367 cluster suppresses the proliferation of cervical carcinoma cells through the novel target AKT1. RNA 19: 85-95. http://dx.doi.org/10.1261/rna.035295.112

Campayo M, Navarro A, Viñolas N, Diaz T, et al. (2013). Low miR-145 and high miR-367 are associated with unfavourable prognosis in resected nonsmall cell lung cancer. Eur. Respir. J. 41: 1172-1178. http://dx.doi. org/10.1183/09031936.00048712

Chae YS, Kim JG, Kang BW, Lee SJ, et al. (2013). Functional polymorphism in the MicroRNA-367 binding site as a prognostic factor for colonic cancer. Anticancer Res. 33: 513-519.

Guan Y, Chen L, Bao Y, Qiu B, et al. (2015). High miR-196a and low miR-367 cooperatively correlate with unfavorable prognosis of high-grade glioma. Int. J. Clin. Exp. Pathol. 8: 6576-6588.

Kaid C, Silva PB, Cortez BA, Rodini CO, et al. (2015). miR-367 promotes proliferation and stem-like traits in medulloblastoma cells. Cancer Sci. 106: 1188-1195. http://dx.doi.org/10.1111/cas.12733

Kanekura K, Nishi H, Isaka K and Kuroda M (2016). MicroRNA and gynecologic cancers. J. Obstet. Gynaecol. Res. 42: 612-617. http://dx.doi.org/10.1111/jog.12995

Lee E, Ito K, Zhao Y, Schadt EE, et al. (2016). Inferred miRNA activity identifies miRNA-mediated regulatory networks underlying multiple cancers. Bioinformatics 32: 96-105.

Ma YB, Song DW, Nie RH and Mu GY (2016). MicroRNA-32 functions as a tumor suppressor and directly targets EZH2 in uveal melanoma. Genet. Mol. Res. 15: 15. http://dx.doi.org/10.4238/gmr.15027935

Meng X, Lu P and Fan Q (2016). miR-367 promotes proliferation and invasion of hepatocellular carcinoma cells by negatively regulating PTEN. Biochem. Biophys. Res. Commun. 470: 187-191. http://dx.doi.org/10.1016/j.bbrc.2016.01.025

Shenouda SK and Alahari SK (2009). MicroRNA function in cancer: oncogene or a tumor suppressor? Cancer Metastasis Rev. 28: 369-378. http://dx.doi.org/10.1007/s10555-009-9188-5

Sun L, Bian G, Meng Z, Dang G, et al. (2015a). miR-144 inhibits uveal melanoma cell proliferation and invasion by regulating c-Met expression. PLoS One 10: e0124428. http://dx.doi.org/10.1371/journal.pone.0124428

Sun L, Wang Q, Gao X, Shi D, et al. (2015b). MicroRNA-454 functions as an oncogene by regulating PTEN in uveal melanoma. FEBS Lett. 589 (19 Pt B): 2791-2796. http://dx.doi.org/10.1016/j.febslet.2015.08.007

Sun J, Song K, Feng X and Gao S (2016). MicroRNA-367 is a potential diagnostic biomarker for patients with esophageal squamous cell carcinoma. Biochem. Biophys. Res. Commun. 473: 363-369. http://dx.doi.org/10.1016/j. bbrc.2016.01.042

Wang GC, He QY, Tong DK, Wang CF, et al. (2016). MiR-367 negatively regulates apoptosis induced by adriamycin in osteosarcoma cells by targeting KLF4. J Bone Oncol 5: 51-56. http://dx.doi.org/10.1016/j.jbo.2016.02.002

Yan D, Zhou X, Chen X, Hu DN, et al. (2009). MicroRNA-34a inhibits uveal melanoma cell proliferation and migration through downregulation of c-Met. Invest. Ophthalmol. Vis. Sci. 50: 1559-1565. http://dx.doi.org/10.1167/iovs.08-2681

Yousef YA and Alkilany M (2015). Characterization, treatment, and outcome of uveal melanoma in the first two years of life. Hematol. Oncol. Stem Cell Ther. 8: 1-5. http://dx.doi.org/10.1016/j.hemonc.2014.09.004

Zhang L, Liu Y, Song F, Zheng H, et al. (2011). Functional SNP in the microRNA-367 binding site in the 3'UTR of the calcium channel ryanodine receptor gene 3 (RYR3) affects breast cancer risk and calcification. Proc. Natl. Acad. Sci. USA 108: 13653-13658. http://dx.doi.org/10.1073/pnas.1103360108

Zhu Z, Xu Y, Zhao J, Liu Q, et al. (2015). miR-367 promotes epithelial-to-mesenchymal transition and invasion of pancreatic ductal adenocarcinoma cells by targeting the Smad7-TGF- $\beta$ signalling pathway. Br. J. Cancer 112: 13671375. http://dx.doi.org/10.1038/bjc.2015.102

Genetics and Molecular Research 16 (3): gmr16039067 